\title{
La red de apoyo familiar y las relaciones intrafamiliares como predictoras de la satisfacción vital
}

\author{
The Family Support Network and Intra-family Relationships \\ as Predictors of Life Satisfaction
}

\author{
Arturo Barraza Macías ${ }^{1}$ \\ https://orcid.org/0000-0001-6262-0940
}

${ }^{1}$ Departamento de Investigación y Posgrado, Universidad Pedagógica de Durango, México

Resumen. Objetivo. Determinar la relación entre la red de apoyo familiar y las relaciones intrafamiliares con la satisfacción vital. Método. Se realizó un estudio correlacional, transversal y no experimental con una muestra de 314 estudiantes de dos instituciones de educación superior: 70 hombres (22.3\%) y 244 mujeres (77.7\%), con una edad promedio de 27.55 años (DT = 9.57). Resultados. Las variables predictoras de la satisfacción vital del individuo fueron: la cantidad de miembros de la red de apoyo familiar; la cercanía o intimidad de contacto entre esos miembros; además de la unión y apoyo y de la expresión mostradas en las relaciones intrafamiliares.

Palabras clave. Satisfacción vital, familia, red de apoyo, universitarios

Abstract. Objective. The main objective of this paper was to determine the relationship between the elements of family support network and intra-family relationships with life satisfaction. Method. A correlational, cross-sectional, and non-experimental study was carried out with a sample of 314 students from two higher education institutions: 70 men (22.3\%) and 244 women (77.7\%), with an average age of 27.55 years ( $S D=9.57$ ). Results. Results showed that the model constituted by the members of the family support network, the closeness or intimacy among those members, the support shown in intra-family relationships, and the communication excel to predict the vital satisfaction of the individual.

Keywords. Life satisfaction, family, support network, university students

${ }^{1}$ Arturo Barraza Macías. Universidad Pedagógica de Durango, Departamento de Investigación y Posgrado. Dirección postal: Josefa Ortiz de Domínguez No. 104, Fraccionamiento Francisco Sarabia, Durango, Dgo. México. C.P. 34214 - Durango, Dgo. - México. E-mail: praxisredie2@gmail.com 


\section{Introducción}

El interés por el estudio del bienestar y otros constructos similares, como la felicidad y la satisfacción vital, ha sido reivindicado por la psicología positiva; sin embargo, este creciente interés de los últimos años no compensa el olvido al que estuvieron estos temas expuestos durante décadas.

La psicología, en lo general, y la psicología clínica, en lo particular, hicieron de lo patogénico su leitmotiv. El avance en el estudio de variables como depresión, ansiedad y estrés postraumático, por mencionar solo algunos ejemplos, ha sido evidente, mientras que el descuido de las variables positivas se ha manifestado en corrientes teóricas disímbolas, términos diversos para un mismo fenómeno, instrumentos de medición con propiedades psicométricas pobres y la yuxtaposición de ámbitos de estudio, todos ellos indicadores de un campo de estudio en proceso de construcción.

En el caso de la satisfacción vital, que es la variable de interés para el presente trabajo, lo primero que hay que discutir es su ubicación teórico conceptual; algunos autores la consideran como parte del Bienestar Subjetivo (Diener et al., 2002), de la Calidad de Vida (Veenhoven, 1994) o de la Economía de la Felicidad (Orozco, 2014).

Para efectos de este trabajo, se considera a la satisfacción vital como el componente cognitivo del Bienestar Subjetivo (Diener, 2000), y es bajo esta línea discursiva que Diener et al. (1985) definen la satisfacción vital como un juicio de carácter cognitivo en que la persona evalúa su vida como una totalidad. Este juicio implica la valoración global del sujeto sobre sus condiciones de vida o de aspectos particulares de esta (Diener et al., 1999) a partir de un criterio estándar o una expectativa que la persona ha construido previamente y considera apropiado.

Ante esta definición de la satisfacción vital, cabe preguntarse ¿qué es lo que influye para que se dé esa evaluación global? Las investigaciones al respecto han abordado tanto aspectos individuales como sociales.

Los estudios sobre los aspectos individuales muestran una alta dispersión de las variables indagadas; lo mismo se estudia con relación a la empatía cognitiva (Reina \& Oliva, 2015) que con la responsabilidad, el optimismo y la extraversión (Cazalla-Luna \& Molero, 2018). Así mismo, se ha estudiado ciertas características de la personalidad como el Neuroticismo y la Amabilidad (Schoeps et al., 2014) y la Inteligencia emocional (García et al., 2020).

En los aspectos sociales, se ha constatado que el apoyo social (Diez et al., 2016; Fernández et al., 2010; Martos, 2016; Novoa \& Barra, 2015), la participación social (Pinazo-Hernandis et al., 2019), la conducta prosocial (Veloso et al., 2015) y la clase social percibida (Vallejo-Martín \& Moreno, 2016) generan un mayor nivel de satisfacción vital. Como se puede observar, el apoyo social se ha mostrado consistente como una variable predictora de la satisfacción vital, además de que los investigadores empiezan a interesarse por otras variables de tipo social.

Más allá del estudio de estas variables sociales, los antecedentes de investigación muestran el interés de los investigadores en un nuevo ámbito: el familiar. Con respecto a esta variable, trabajos previos han mostrado que el apoyo familiar (Hombrados-Mendieta et al., 2016), la consistencia familiar permisiva (Torre et al., 2013), el autoconcepto familiar (Casadiego et al., 2018) y la valoración sobre la cohesión familiar (Schoeps et al., 2014) se correlacionan positivamente con la satisfacción vital, mientras que la disfunción familiar (Nie et al., 2020), el estrés parental (Melguizo-Garín et al., 2019) y el estrés familiar (Collantes, 2018) se correlacionan negativamente. 
No obstante, este creciente interés por el ámbito familiar evidencia la multiplicidad de variables abordadas y la falta de continuidad, o ausencia, de líneas claras de investigación. En ese sentido, es necesario recordar que la familia es considerada como un conjunto organizado e interdependiente de individuos (red de apoyo familiar) que mantienen una constante interacción (relaciones intrafamiliares), que suele ser regulada por reglas y por funciones dinámicas de carácter sistémico que permiten la coexistencia interna y con el exterior (Minuchin, 1986). Para ampliar la investigación en este campo, el presente estudio aborda dos variables propias del ámbito familiar: la red de apoyo familiar y las relaciones intrafamiliares.

En el caso de la primera, se realiza un recorte empírico objetual de la variable del apoyo social y se circunscribe al ámbito familiar para indagar la red de apoyo familiar tanto en su dimensión estructural, que indica la cantidad de personas que conforman esa red, como en su dimensión funcional, que incluye la frecuencia de contacto y la cercanía/ intimidad de las relaciones.

Villafrade y Franco (2016) abordan el estudio de la familia como red de apoyo social, y establecen entre sus características identitarias el tiempo de convivencia (frecuencia de contacto) y los vínculos entre padres e hijos (cercanía/intimidad de las relaciones); sin embargo, a pesar de existir estudios previos sobre este tema (Contreras \& Hernández, 2019; Matos et al., 2019), no se localizó algún trabajo que lo relacionara con la satisfacción vital.

En el caso de la segunda variable, se estudian las relaciones intrafamiliares como interconexiones que se dan entre los integrantes de una familia (Rivera \& Andrade, 2010); su estudio se aborda a partir de tres de sus características centrales: (a) la unión y el apoyo que conduce a sus integrantes a convivir y a realizar actividades en conjunto; (b) la expresión, que se manifiesta en la posibilidad de comunicar las emociones, ideas y acontecimientos; (c) y las dificultades que implica la percepción de la situaciones problemáticas o negativas de la familia.

Las relaciones intrafamiliares se han estudiado desde variables como la dependencia emocional (Mallma, 2016), las conductas violentas (Bonilla et al., 2017), la calidad de vida relacionada con la salud (Chávez-Flores et al., 2018), la satisfacción familiar (Lagos, 2019) y el estrés cotidiano (Ruiz, 2019), por mencionar solamente algunas. Cabe destacar que, a pesar de no haber localizado algún estudio que aborde su relación con la variable satisfacción vital, sí se pudo localizar trabajos que la relacionan con el bienestar subjetivo que, como se mencionó anteriormente, integra a la satisfacción vital como uno de sus componentes.

En estas investigaciones, se encontró que las relaciones intrafamiliares se correlacionan de manera positiva y débil con el bienestar subjetivo (Matus, 2017; Zaquinaula \& Murillo, 2015), mostrando solamente diferencias en la dimensión de dificultades, ya que mientras Matus (2017) afirma que existe relación con el bienestar subjetivo, Zaquinaula y Murillo (2015) lo niegan. Estos antecedentes justifican la creciente necesidad de estudiar la relación de esta variable con la satisfacción vital.

Con base en estos antecedentes, se plantea como objetivo de la presente investigación determinar la relación que existe entre la red de apoyo familiar y las relaciones intrafamiliares con la satisfacción vital en estudiantes de educación superior. 


\section{Método}

\section{Participantes}

El presente estudio de carácter correlacional, se realizó con una muestra de 314 estudiantes de dos instituciones de educación superior de la ciudad de Durango, en México. Esta muestra fue de tipo no probabilística y estuvo determinada por la accesibilidad a las personas encuestadas. Los criterios de inclusión fueron ser estudiante de educación superior, haber asistido a clases el día que se aplicó el cuestionario y tener disponibilidad y disposición para responderlo.

Tabla 1

Caracterización de la muestra

\begin{tabular}{|c|c|c|}
\hline Variable & $n$ & $\%$ \\
\hline \multicolumn{3}{|l|}{ Sexo } \\
\hline Hombre & 70 & 22.3 \\
\hline Mujer & 244 & 77.7 \\
\hline \multicolumn{3}{|l|}{ Institución } \\
\hline Universidad Pedagógica de Durango & 223 & 71 \\
\hline Universidad Autónoma de Durango & 91 & 29 \\
\hline \multicolumn{3}{|l|}{ Nivel de estudios } \\
\hline Licenciatura & 245 & 78 \\
\hline Posgrado & 69 & 22 \\
\hline \multicolumn{3}{|l|}{ Semestre } \\
\hline \multicolumn{3}{|l|}{ Licenciatura } \\
\hline Primero & 43 & 18.1 \\
\hline Segundo & 0 & 0 \\
\hline Tercero & 43 & 18.1 \\
\hline Cuarto & 22 & 9.2 \\
\hline Quinto & 79 & 33.3 \\
\hline Sexto & 0 & 0 \\
\hline Séptimo & 46 & 19.4 \\
\hline Octavo & 1 & .4 \\
\hline Noveno & 3 & 1.2 \\
\hline \multicolumn{3}{|l|}{ Posgrado } \\
\hline Primero & 10 & 14.4 \\
\hline Segundo & 1 & 1.4 \\
\hline Tercero & 37 & 53.6 \\
\hline Cuarto & 1 & 1.4 \\
\hline Quinto & 19 & 27.5 \\
\hline Sexto & 1 & 1.4 \\
\hline
\end{tabular}

Nota. En el caso del semestre, existía un 2.5\% de valores perdidos, por lo que se utilizó el porcentaje válido y en la suma total de $n$ faltan los ocho valores perdidos. 
La distribución de las personas encuestadas según las variables sociodemográficas indagadas en la sección de datos personales se presenta en la Tabla 1. Como se puede observar en esta muestra, hay una preponderancia de mujeres que cursan el nivel de licenciatura en el quinto semestre. Con respecto a la edad, el valor mínimo fue de 18 años y el máximo de 57 años, siendo el promedio 27.55 años $(D T=9.57)$.

\section{Instrumentos}

Para la recolección de la información, se utilizó un cuestionario compuesto de cuatro secciones. (a) En la sección uno, se indagan los datos personales: sexo, edad, institución educativa, nivel educativo y semestre que se cursaba; (b) la sección dos está constituida por el Cuestionario de Satisfacción Vital (Diener et al., 1985); (c) la sección tres la conforman seis ítems que indagan los factores de frecuencia, cantidad y cercanía con dos grupos familiares: la familia de origen y los parientes cercanos; (d) y la sección cuatro se conforma por la Escala Breve de Evaluación de Relaciones Intrafamiliares (Rivera \& Andrade, 2010).

El Cuestionario de Satisfacción Vital, en su versión original, fue elaborado por Diener et al. (1985), pero en esta investigación se utilizó la traducción ofrecida por Seligman (2003). En esta versión, se mantiene sin cambios la versión original con cinco reactivos redactados en forma afirmativa, cada uno con siete posibilidades de respuesta que van desde (1) muy en desacuerdo a (7) muy de acuerdo.

En el momento de su aplicación, el cuestionario de Satisfacción Vital obtuvo un coeficiente de confiabilidad de $\alpha$.81. Así mismo, el Análisis Factorial Exploratorio, realizado a través del método de mínimos cuadrados no ponderados con rotación oblimin directo, arroja un solo factor que explica el 52\% de la varianza. Este análisis arrojó los siguientes resultados: (a) $K M O=.84$; (b) nivel de significación de la prueba de esfericidad de Bartlett es de $p<.001$; (c) cuatro ítems con comunalidades superiores a .30, salvo el ítem cinco que reporta una comunalidad extraída de .24; (d) todos los ítems saturan en el factor único siendo su valor menor de .49, correspondiente al ítem cinco.

En el Análisis Factorial Confirmatorio se utilizó el método de estimación de máxima verosimilitud, se obtuvieron los siguientes resultados: $\chi 2=8.21$; $d f=5$; valores de $p=$ $0.14 ; \times 2 / d f=1.6 ; G F I=0.99 ; A G F I=0.97 ; R M S E A=0.04 ;$ Intervalo de confianza para $R M S E A=.00-.09 ;$ el valor de $p$ en la prueba para el ajuste perfecto $(R M S E A<.05)=.49$; $C F I=1.00$, e Independencia $A / C=845.43$. Estos indicadores muestran un buen ajuste del modelo ya que:

- En el caso de valores de $p$ de la $\chi 2$ se considera que valores $>.05$ indican un buen ajuste de los datos (Ruiz et al., 2010).

- En lo que respecta a $\chi 2 / d f$ se plantea que valores inferiores a 2 indican un buen ajuste (Escobedo et al., 2016).

- Por su parte, en el RMSEA, se señala que hay un ajuste aceptable cuando sus valores son inferiores a .05 (Ruiz et al., 2010).

- En el caso de GFI se indica que valores mayores a .90 representan un ajuste óptimo (Escobedo et al., 2016).

- En lo que respecta a AGFI los valores mayores o iguales a .95 son los que muestran un mejor ajuste del modelo (Ruiz et al., 2010).

- En lo que respecta al CFI, los valores de .95 o superiores muestran un ajuste óptimo (Ruiz et al., 2010). 
Los ítems que conformaban la sección tres se distribuían de la siguiente manera: (a) dos ítems preguntaban con qué frecuencia convivían o se relacionaban con su familia de origen (padre, madre, hermanos y hermanas) y con sus parientes cercanos (abuelos, abuelas, tíos, tías, primos y primas). Para responder, se les ofreció un escalamiento de frecuencia de cuatro valores: nunca, algunas veces, casi siempre y siempre; (b) los siguientes dos ítems preguntaban con cuántas personas se relacionaban habitualmente de su familia de origen y sus parientes cercanos. Para responder, se les dio cuatro opciones: de uno a tres, de cuatro a seis, de siete a nueve y de diez en adelante; (c) y las últimas dos preguntas indagaban la cantidad de confianza, intimidad o cercanía que existe con aquellas personas con las que se relacionan dentro de su familia de origen y sus parientes cercanos. Para responder se les proporcionó un escalamiento de cuatro valores en el que uno era poco y cuatro era mucho.

La Escala de Evaluación de Relaciones Intrafamiliares, en sus tres versiones, larga, intermedia y breve, fue diseñada y validada por Rivera y Andrade (2010). La versión breve, que es la utilizada en la presente investigación, consta de 12 ítems distribuidos en tres dimensiones: (a) unión y apoyo (ítems 1, 4, 7, 10); (b) expresión (ítems 2, 5, 8, 11); (c) y dificultades (ítems 3, 6, 9, 12). Cada uno de los ítems tiene cinco opciones de respuesta que van desde: totalmente de acuerdo a totalmente en desacuerdo; en esta ocasión, se eliminaron los valores o categorías verbales intermedias, y solamente, se les ofreció un escalamiento de cinco números (del 1 al 5). Además, se les indicó que el 1 era "total desacuerdo" y el 5, "total acuerdo". Al eliminar la opción de respuesta "neutral (ni de acuerdo ni en desacuerdo)" se evitó el fenómeno de regresión a la media.

En esta aplicación la Escala Breve de Evaluación de Relaciones Familiares obtuvo un coeficiente de confiabilidad de $\alpha .86$. Así mismo, el Análisis Factorial Exploratorio arroja tres factores que explican el $54 \%$ de la varianza. En este punto, cabe mencionar que originalmente el programa arroja una solución de dos factores, que integra las dimensiones de unión y apoyo y expresión en una sola; no obstante, este resultado se le solicitó al programa tres factores y sus resultados son los que se presentan en esta ocasión.

El Análisis Factorial Exploratorio, realizado a través del método de mínimos cuadrados no ponderados con rotación oblimin directo, presentó los siguientes resultados: (a) KMO = 869; (b) nivel de significación de la prueba de esfericidad de Bartlett es de $p=.001$; (c) diez ítems con comunalidad superiores a .3, salvo los ítem tres y nueve que reportan una comunalidad extraída de 25 y .29, respectivamente; (d) todos los ítems saturan en el factor que les corresponde a excepción del ítem cuatro que satura en el factor uno correspondiente a la dimensión de expresión, siendo su ubicación correcta la dimensión unión y apoyo. En todos los casos se obtuvieron saturaciones superiores a .35, siendo su valor menor de .52, correspondiente al ítem nueve.

En el Análisis Factorial Confirmatorio, en el que se utilizó el método de estimación de máxima verosimilitud, se obtuvieron los siguientes resultados: $\chi 2=181.29 ; d f=51$; valor de $p=.00 ; \chi 2 / d f=3.55 ; \mathrm{GFI}=.91 ; \mathrm{AGFI}=.87 ; \mathrm{RMSEA}=.09 ;$ Intervalo de confianza para $R M S E A=.76-.10$; el valor de $p$ en la prueba para el ajuste perfecto $(R M S E A<.05)=.00$; $C F I=0.96$, e Independencia $A / C=3447.83$. Como se muestra, el modelo de tres factores presenta algunos problemas:

- En el valor de $p$ de la $\chi 2$, se considera que valores > .05 indican un buen ajuste de los datos, y, en este caso, se obtuvo .00 .

- En lo que respecta a $\chi 2 / d f$, se plantea que valores inferiores a 2 indican un buen ajuste, $y$, en este caso, se obtuvo 3.55. 
- En el RMSEA se señala que hay un ajuste aceptable cuando sus valores son inferiores a .05, y, en este caso, se obtuvo 09 .

- En lo que respecta a AGFI, los valores mayores o iguales a .95 son los que muestran un mejor ajuste del modelo, y, en este caso, se obtuvo 87 .

Estos datos demuestran problemas con su dimensionalización o estructura interna; sin embargo, no es el objetivo de la presente investigación hacer la adecuación de la escala en ese aspecto por lo que solamente se informan los resultados y se utiliza la versión tal y como es reportada por los autores de la misma.

\section{Procedimiento}

Una vez que se tuvo el cuestionario conformado por las cuatro secciones, se procedió a su aplicación en una muestra de estudiantes de dos instituciones de educación superior. En primer lugar, se procedió a hablar con los directivos de cada institución para explicarles el objetivo de la investigación y asegurarles que no existía ningún riesgo para las personas encuestadas.

Al obtener la autorización de los directivos de cada una de las dos instituciones, se procedió a la aplicación del cuestionario explicándoles a los estudiantes el carácter voluntario de su respuesta. En ninguno de los casos hubo necesidad de explicar algo más, ya que todos decidieron participar. Cabe mencionar que no se les pidió la autorización por escrito, pero sí se les hizo énfasis y se dejó perfectamente en claro que, si aceptaban responder el cuestionario, consentían participar en el estudio. La aplicación se dio en las propias aulas donde los estudiantes cursan sus estudios, con la previa autorización del maestro que en ese momento se encontraba laborando con ellos.

Una vez que se aplicó el cuestionario, se construyó la base de datos en el programa SPSS versión 23. En seguida, se realizó la imputación de los valores perdidos tomando como métodos la media de la serie y la mediana de puntos cercanos, según correspondiera al nivel de medición de los ítems. Posteriormente, se calcularon las dimensiones y variables correspondientes en cada caso.

\section{Análisis de datos}

Una vez que se tuvo conformada la versión final de la base de datos, se procedió a hacer los siguientes análisis en el programa SPSS, versión 23: (a) se realizó el análisis de las propiedades psicométricas de los instrumentos utilizados a través del estadístico alfa de Cronbach y, en el Análisis Factorial Exploratorio, se utilizó el método de mínimos cuadrados no ponderados con rotación oblimin directo; (b) se importó la base de datos al programa LISREL 8.80 Student, y se realizó en el mismo el Análisis Factorial Confirmatorio utilizando el método de estimación de máxima verosimilitud; (c) se obtuvieron los datos descriptivos de cada ítem de las tres variables a partir de la media y la desviación típica; (d) se efectuó el análisis correlacional de las variables de red de apoyo familiar y relaciones intrafamiliares, así como de sus respectivas dimensiones con la variable satisfacción vital a partir del estadígrafo $r$ de Pearson $(p<.05)$; (e) y se realizó el Análisis de Regresión Lineal Múltiple mediante el método paso a paso en dos modelos:

1. Red de apoyo familiar más relaciones intrafamiliares;

2. Cantidad de miembros de la red de apoyo familiar más la cercanía o intimidad de contacto con esos miembros, más la unión y apoyo mostrado en las relaciones intrafamiliares, más la expresión mostrada en las relaciones intrafamiliares. 
La decisión de utilizar un modelo con variables y otro con dimensiones se debe a que el análisis correlacional previo mostró que, en cada variable, había una dimensión que mostraba una correlación muy baja con la variable satisfacción vital. En el caso de la red de apoyo social, era la dimensión de frecuencia de contacto y, en el de las relaciones intrafamiliares, era la dimensión de dificultades. Por esto, ambas fueron eliminadas de este segundo análisis.

En el caso del primer modelo, se cubrieron los cinco supuestos básicos: linealidad (los gráficos de regresión parcial mostraron asociación entre las variables), independencia (DW: 1.97), homocedasticidad (significación de la prueba de Levene para satisfacción vital de .05 , para relaciones intrafamiliares de .49 y para la red de apoyo social de .69), normalidad $(K S=.07$ para satisfacción vital y $K S=.09$ para para relaciones intrafamiliares y la red de apoyo social) y no colinealidad (tolerancia de .87 para ambos casos; y VIF de 1.40 para ambos casos).

En el segundo modelo, se cubrieron los cinco supuestos básicos: linealidad (los gráficos de regresión parcial mostraron asociación entre las variables), independencia ( $D W: 1.97)$, homocedasticidad (significación de la prueba de Levene para satisfacción vital de .05, para la cantidad de miembros de la red de apoyo familiar de .01, para la cercanía o intimidad de contacto con los miembros de la red de .900, para la unión y apoyo mostrado en las relaciones intrafamiliares de .99 y para la expresión mostrada en las relaciones intrafamiliares de .76), normalidad ( $K$-S entre .07 y .09 para los diferentes casos) y no colinealidad (tolerancia entre .48 y .97 para los diferentes casos; y VIF entre 1.02 y 1.95 para los diferentes casos).

\section{Consideraciones éticas}

El proyecto que generó la presente investigación fue aprobado por el Comité de Investigación de la institución de adscripción del autor. Es menester reconocer que, en esta institución, no existe un comité de ética que valore este aspecto en lo específico; sin embargo, el Comité de Investigación Institucional valora tanto los aspectos metodológicos como los éticos de los proyectos de investigación.

La autorización de este proyecto, en los aspectos éticos, se dio con apego a la protección de los derechos y garantías de los participantes en la investigación según la sección 8.01 de los principios éticos de la American Psychological Association (2017) y de acuerdo con el artículo 17, fracción I, del Reglamento de la Ley General de Salud en Materia de Investigación en Salud (Secretaría de Salud, 1987), que considera que este tipo de investigación se debe tomar como una sin riesgo, ya que, en la recolección de la información, solo se utilizaron cuestionarios que no afectaron ni generaron cambios, de manera intencionada o deliberada, en variables psicológicas y/o sociales de los participantes.

El cuestionario tuvo un carácter anónimo y, en la presentación del mismo, se les aseguró la confidencialidad de los resultados; con relación al consentimiento informado, se les enfatizó el carácter voluntario de su llenado haciéndoles ver que el hecho de responderlo era que consentían participar en dicha investigación. Así mismo, no se les solicitó ningún dato sociodemográfico que comprometiera su anonimato.

\section{Resultados}

Los datos descriptivos de los ítems de las tres variables indagadas se presentan en la Tabla 2. Como se puede observar, en el caso de la satisfacción vital, los ítems que se presentan con mayor frecuencia son el tres, "Estoy completamente satisfecho con mi vida", y el cuatro, "Hasta ahora, he conseguido las cosas que para mí son importantes en la vida". 
Por su parte, los ítems de la red de apoyo familiar muestran que la población encuestada casi siempre convive o se relaciona con su familia de origen y que la confianza, intimidad o cercanía que existe con esta es cuantiosa; en el caso de las relaciones intrafamiliares, los datos muestran que las personas encuestadas consideran que su familia es cálida y les brinda apoyo, y que la atmósfera familiar usualmente no es desagradable.

Tabla 2

Datos descriptivos de los items que conforman las tres variables indagadas $(n=374)$

\begin{tabular}{|c|c|c|}
\hline Variables & M & DT \\
\hline \multicolumn{3}{|l|}{ Satisfacción vital } \\
\hline En la mayoría de los aspectos, mi vida se acerca a mi ideal. & 5.18 & 1.39 \\
\hline Las condiciones de mi vida son excelentes. & 5.15 & 1.31 \\
\hline Estoy completamente satisfecho con mi vida. & 5.42 & 1.34 \\
\hline Hasta ahora, he conseguido las cosas que para mí son importantes en la vida. & 5.45 & 1.44 \\
\hline Si pudiera vivir de nuevo, no cambiaría nada. & 4.77 & 1.97 \\
\hline \multicolumn{3}{|l|}{ Red de apoyo familiar } \\
\hline $\begin{array}{l}\text { Frecuencia con la que conviven o se relacionan con su familia de origen (padres, } \\
\text { madres, hermanos y hermanas). }\end{array}$ & 3.21 & .81 \\
\hline $\begin{array}{l}\text { Frecuencia con la que conviven o se relacionan con sus parientes cercanos } \\
\text { (abuelas, abuelos, tíos, tías, primos y primas). }\end{array}$ & 2.27 & .83 \\
\hline Con cuántas personas se relacionaba habitualmente de familia de origen. & 2.05 & 1.07 \\
\hline Con cuántas personas se relacionaba habitualmente de parientes cercanos. & 2.49 & 1.21 \\
\hline $\begin{array}{l}\text { Qué tanta confianza, intimidad o cercanía existe con las personas con que se } \\
\text { relaciona de su familia de origen. }\end{array}$ & 3.32 & .84 \\
\hline $\begin{array}{l}\text { Qué tanta confianza, intimidad o cercanía existe con las personas con que se } \\
\text { relaciona de sus parientes cercanos. }\end{array}$ & 2.36 & .93 \\
\hline \multicolumn{3}{|l|}{ Relaciones intrafamiliares } \\
\hline Los miembros de la familia acostumbran a hacer cosas juntos. & 3.81 & 1.10 \\
\hline Mis padres me animan a expresar abiertamente mis puntos de vista. & 3.81 & 1.21 \\
\hline En mi familia, nadie se preocupa por los sentimientos de los demás. & 3.69 & 1.46 \\
\hline Mi familia es cálida y nos brinda apoyo. & 4.20 & 1.09 \\
\hline En nuestra familia es importante para todos expresar nuestras opiniones. & 3.93 & 1.19 \\
\hline La atmósfera de mi familia usualmente es desagradable. & 4.05 & 1.28 \\
\hline Nuestra familia acostumbra a hacer actividades en conjunto. & 3.77 & 1.18 \\
\hline Mi familia me escucha. & 3.92 & 1.18 \\
\hline Cuando tengo algún problema no se lo platico a mi familia. & 3.51 & 1.50 \\
\hline Los miembros de la familia de verdad nos ayudamos y apoyamos unos a otros. & 3.93 & 1.20 \\
\hline En mi familia expresamos abiertamente nuestro cariño. & 3.68 & 1.31 \\
\hline Los conflictos en mi familia nunca se resuelven. & 3.80 & 1.31 \\
\hline
\end{tabular}

Nota. Se destacan con negritas las medias más altas. 
Por su parte, los datos descriptivos de las dimensiones y variables indagadas se presentan en la Tabla 3. Estas medias se transformaron en porcentaje a través de la regla de tres simple para presentar los siguientes resultados.

Como se puede observar, el cuerpo estudiantil encuestado presenta un alto nivel de satisfacción vital (74\%) y de características positivas en sus relaciones intrafamiliares (76\%); asimismo, las dimensiones con mayor presencia son la cercanía o intimidad de contacto (71\%), en el caso de la red de apoyo familiar, y la unión y apoyo (78\%), en el caso de las relaciones intrafamiliares. Cabe mencionar que, para la interpretación de estos porcentajes, se estableció un baremo indicativo basado en el valor teórico de la variable con tres puntos de corte: de 0\% a 33\% (valor bajo), de 34\% a 66\% (valor moderado) y de $67 \%$ a $100 \%$ (valor alto).

Tabla 3

Datos descriptivos de las dimensiones y variables indagadas $(n=314)$

\begin{tabular}{lcc}
\hline \multicolumn{1}{c}{ Variables y dimensiones } & $M$ & $D T$ \\
\hline Red de apoyo familiar & 2.62 & .55 \\
Frecuencia de contacto & 2.74 & .70 \\
Cantidad de miembros & 2.27 & .88 \\
Cercanía o intimidad en el contacto & 2.84 & .72 \\
Relaciones intrafamiliares & 3.84 & .80 \\
Unión y apoyo & 3.93 & .96 \\
Expresión & 3.83 & 1.00 \\
Dificultades & 3.76 & 1.01 \\
Satisfacción vital & 5.19 & 1.14 \\
\hline
\end{tabular}

Nota. Se destacan con negritas las medias más altas en cada variable.

Tabla 4

Coeficiente de correlación entre las variables y dimensiones de la red de apoyo familiar y relaciones intrafamiliares con la variable satisfacción vital $(n=314)$

\begin{tabular}{lc}
\hline Variable y dimensiones & Satisfacción vital \\
\hline Red de apoyo familiar & $.34^{\star *}$ \\
Frecuencia de contacto & $.13^{\star}$ \\
Cantidad de miembros & $.26^{\star *}$ \\
Cercanía o intimidad en el contacto & $.30^{\star *}$ \\
Relaciones intrafamiliares & $.37^{\star *}$ \\
Unión y apoyo & $.36^{\star *}$ \\
Expresión & $.39^{\star *}$ \\
Dificultades & $.19^{\star *}$ \\
\hline
\end{tabular}

${ }^{\star} p<.05 .{ }^{* *} p<.001$. 
Los resultados del análisis correlacional entre las variables y dimensiones de la red de apoyo familiar y relaciones intrafamiliares con la variable satisfacción vital se presentan en la Tabla 4. Como se muestra, tanto las variables como sus dimensiones se relacionan de manera positiva con la satisfacción vital; por lo contrario, se observa que las dimensiones frecuencia de contacto y dificultades presentan las correlaciones más débiles. En ese sentido, se decide trabajar con dos modelos diferentes para el análisis de regresión lineal múltiple: (a) la variable red de apoyo familiar junto a la de las relaciones intrafamiliares como predictoras de la satisfacción vital; ambas se calculan con base en sus tres dimensiones; (b) y la dimensión cantidad de miembros junto a la dimensión cercanía o intimidad en el contacto más la dimensión unión y apoyo más la expresión como predictoras de la satisfacción vital.

En el análisis de regresión lineal del primer modelo, los resultados indican un valor para $R^{2}$ de .10 y .17, respectivamente, lo que indica que el modelo en total explica el $17 \%$ de la variación del nivel de satisfacción vital de la población encuestada (Tabla 5). En el análisis de regresión lineal del segundo modelo, los resultados muestran un valor para $R^{2}$ de $.06, .12, .18$ y .19 respectivamente, lo que indica que el modelo en total explica $19 \%$ de la variación del nivel de satisfacción vital de las personas encuestadas (Tabla 5).

Tabla 5

Modelos de regresión lineal múltiple

\begin{tabular}{|c|c|c|c|c|c|}
\hline \multirow[b]{2}{*}{ Modelos } & \multicolumn{3}{|c|}{ Resumen del modelo } & \multirow{2}{*}{$\begin{array}{c}\text { Prueba global del modelo } \\
F\end{array}$} & \multirow[b]{2}{*}{$\beta$} \\
\hline & $R$ & $R^{2}$ & $\begin{array}{l}\text { Error estándar } \\
\text { de la estimación }\end{array}$ & & \\
\hline $\begin{array}{l}\text { Primer modelo } \\
\text { (RAP) }\end{array}$ & .32 & .10 & 1.08 & $36.84^{\star \star}$ & $.32^{\star *}$ \\
\hline $\begin{array}{l}\text { Primer modelo } \\
(\mathrm{RAP}+\mathrm{RI})\end{array}$ & .41 & .17 & 1.04 & $31.94^{\star \star}$ & $.27^{\star *}$ \\
\hline $\begin{array}{l}\text { Segundo modelo } \\
\text { (CMR) }\end{array}$ & .25 & .06 & 1.11 & $22.49 * *$ & $.25^{\star *}$ \\
\hline $\begin{array}{l}\text { Segundo modelo } \\
(C M E+I C)\end{array}$ & .35 & .12 & 1.07 & $22.95^{\star *}$ & $.25^{\star *}$ \\
\hline $\begin{array}{l}\text { Segundo modelo } \\
(C M R+I C+U y A)\end{array}$ & .42 & .18 & 1.04 & $23.17^{* *}$ & $.25^{\star *}$ \\
\hline $\begin{array}{l}\text { Segundo modelo } \\
(C M R+I C+U y A+E)\end{array}$ & .44 & .19 & 1.03 & $19.12^{* *}$ & $.17^{\star}$ \\
\hline
\end{tabular}

Nota . RAP = Red de Apoyo Familiar; $\mathrm{RI}=$ Relaciones Intrafamiliares; $\mathrm{CMR}=$ Cantidad de miembros de la Red; IC = Intimidad de Contacto; UyA = Unión y Apoyo; E = Expresión.

${ }^{*} p<.05 .{ }^{* *} p<.001$

\section{Discusión}

El análisis efectuado permite concluir que el modelo conformado por la cantidad de miembros de la red de apoyo familiar, más la cercanía o intimidad de contacto con esos miembros, más la unión y apoyo mostrado en las relaciones intrafamiliares, más la expresión y comunicación mostrada en las relaciones intrafamiliares predice en un 19\% la variación del nivel de satisfacción vital del individuo. 
Este resultado conduce, en primera instancia, a analizar los problemas de conceptualización de varios de los constructos presentes en el estudio de la familia, tales como la cohesión familiar, la funcionalidad familiar, la estructura familiar, la dinámica familiar, la comunicación familiar, la socialización familiar, etc., siendo las dos variables analizadas parte de esa problemática. En el caso de la presente investigación, se encontró que el constructo red de apoyo familiar no está tematizado, discutido o investigado con la seriedad que requiere para conformar un modelo teórico conceptual. Por ello, para su estudio, se asumió una aproximación cualitativa (Villafrade \& Franco, 2016) y dos de las perspectivas clásicas en la investigación del apoyo social (la estructural y la funcional). En términos operativos, se indagaron tres indicadores; sin embargo, los resultados mostraron que solo dos de ellos eran pertinentes en la búsqueda de un modelo que explicara en un mayor porcentaje la satisfacción vital.

Por otra parte, se asumió la variable de las relaciones intrafamiliares a partir de la dimensionalización brindada por Rivera y Andrade (2010); no obstante, al igual que con la variable de la red de apoyo familiar, se encontró que sus tres dimensiones constitutivas no mostraban el mismo nivel de pertinencia para la búsqueda de un modelo que explicara la satisfacción vital.

Ante esta situación, se construye un modelo que integra solo dos dimensiones de la red de apoyo familiar y dos dimensiones de las relaciones intrafamiliares. Pese a esto, los análisis efectuados indican una variabilidad en la importancia de las mismas. En ese sentido, a manera de hipótesis teórica, se plantea un modelo que toma como eje central a la interacción entre las dimensiones de cercanía o intimidad de contacto con los miembros de la red de apoyo familiar y la unión y apoyo mostrado en las relaciones intrafamiliares, constituyéndolas en el núcleo central de la relación con la satisfacción vital, mientras que las dimensiones como la cantidad de miembros de la red de apoyo familiar y la expresión y comunicación mostrada en las relaciones intrafamiliares constituyen insumos que posibilitan la existencia del núcleo central de esta relación (Figura 1).

Esta reconfiguración del modelo en tres dimensiones: núcleo central (cercanía o intimidad de contacto con los miembros de la red de apoyo familiar más la unión y apoyo mostrado en las relaciones intrafamiliares), más cantidad de miembros de la red de apoyo familiar, más la expresión y comunicación mostrada en las relaciones intrafamiliares muestra que el núcleo central explica por sí solo un 15\% de la variación del nivel de satisfacción vital de la población encuestada.

En el caso de la representación gráfica del modelo propuesto, se hace una diferenciación en el papel que juegan las dos dimensiones insumos, por lo que la influencia de la cantidad de miembros de la red se presenta con una línea punteada que figura un menor nivel en el núcleo central $\left(r=.22^{\star *}\right)$, mientras que a la expresión y comunicación se le presenta con una fecha sólida que indicaría mayor influencia sobre el núcleo central $\left(r=.65^{\star \star}\right)$.

Esta reconfiguración del modelo planteado para explicar la satisfacción vital deja varias tareas pendientes. En primer lugar, el núcleo central y sus dos dimensiones constituyentes se asemejan al constructo cohesión familiar que puede ser entendido como el vínculo socioemocional que los integrantes de la familia tienen entre sí. Este vínculo incluye diversos tipos de relaciones como la implicación, las alianzas padreshijos y el tiempo dedicado a la relación o a la toma de decisiones en conjunto (Urbano et al., 2018). En ese sentido, se hace necesaria una clarificación conceptual al respecto que tome como base los indicadores empíricos. 


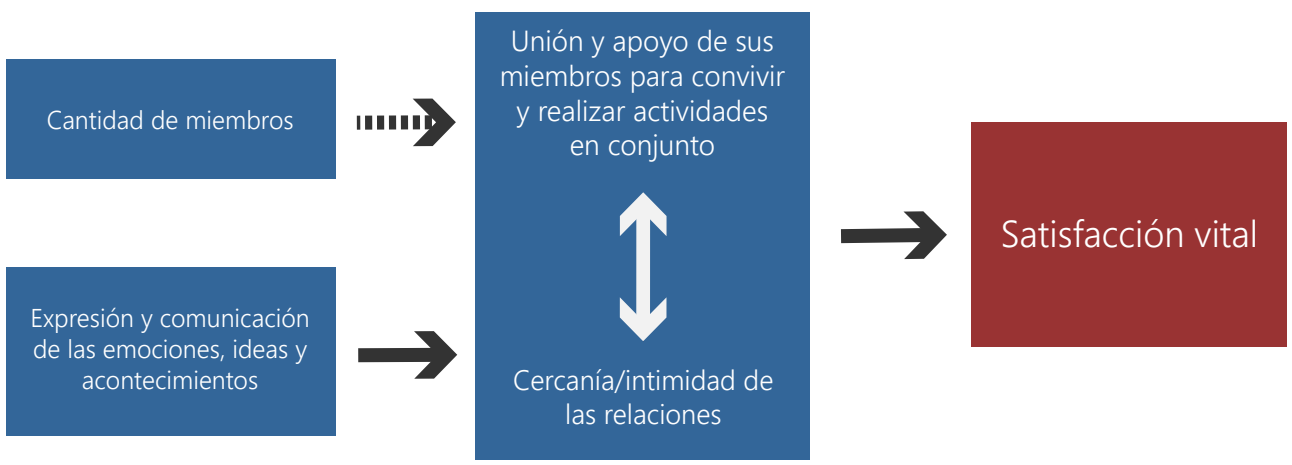

Figura 1. Modelo construido para explicar el rol de las características de la red de apoyo familiar y las relaciones intrafamiliares como predictoras de la satisfacción vital.

En segundo lugar, es necesario que este modelo teórico sea constatado en estudios posteriores en los que se indaguen específicamente estas cuatro dimensiones en relación con la satisfacción vital. Por último, se deberá estudiar si la relación establecida hipotéticamente entre estas cuatro dimensiones es correcta.

Para cerrar este artículo es menester reconocer las principales limitaciones: la composición de la muestra, pues se puede observar que la muestra estuvo compuesta mayoritariamente por mujeres; las propiedades psicométricas de la Escala Breve de Evaluación de Relaciones Intrafamiliares, ya que los análisis efectuados demostraron la presencia de inconsistencias psicométricas, sobre todo en lo referente a la estructura interna de la escala; por último, la debilidad teórico-conceptual de la variable de la red de apoyo familiar, debido a que se estableció que el constructo de red de apoyo familiar no se ha discutido lo suficiente ni conceptual ni teóricamente.

En este último punto, al tomar en consideración que se indagó la red de apoyo familiar con tres indicadores empíricos, se puede esbozar una definición provisional de este concepto en los siguientes términos: la red de apoyo familiar es el conjunto de personas que conforman la familia de origen y los parientes cercanos, los cuales establecen vínculos caracterizados por una mayor o menor frecuencia de contacto y de cercanía o intimidad. Queda en manos de la comunidad investigadora esta definición y la posibilidad de seguir indagando en esta.

\section{Referencias}

American Psychological Association (2017). Ethical principles of psychologists and code of conduct. http://www.apa.org/ethics/code/

Bonilla, C. E., Núñez, S. M., Domínguez, R., \& Callejas, J. E. (2017). Las relaciones intrafamiliares de apoyo como mecanismo explicativo de la conducta violenta en adolescentes escolarizados. Universitas Psychologica, 16(4), 1-12. http://dx.doi. org/10.11144/Javeriana.upsy16-4.riam 
Cazalla-Luna, N., \& Molero, D. (2018). Emociones, afectos, optimismo y satisfacción vital en la formación inicial del profesorado. Profesorado. Revista de currículum y formación del profesorado, 22(1), 215-233. https://recyt.fecyt.es/index.php/ profesorado/article/view/63642/38700

Casadiego, A. M., Trujillo, C., Gaitán, C. E., Chávarro, G. M., Cuervo, L. C., \& Díaz, M. L. (2018). Factores predictores de la satisfacción vital en estudiantes de educación de la Universidad Surcolombiana. Actualidades en Psicología, 32(124), 1-13. http:// dx.doi.org/10.15517/ap.v32i124.26780

Chávez-Flores, Y. V., Hidalgo-Rasmussen, C. A., \& Muñoz, S. (2018). Relaciones intrafamiliares y calidad de vida relacionada con la salud en adolescentes mexicanos que se autolesionan sin intención suicida. Salud \& Sociedad, 9(2), 130-144. http://dx.doi. org/10.22199/S07187475.2018.0002.00001

Collantes, A. I. (2018). Estrés familiar y satisfacción vital en estudiantes del $4^{\circ}$ y $5^{\circ}$ grado de secundaria, del turno mañana, del colegio nacional de mujeres "Edelmira del Pando" de Ate Vitarte, 2017 [Tesis de maestría inédita, Universidad Peruana Unión]. Repositorio de la Universidad Peruana Unión. https://repositorio.upeu. edu.pe/bitstream/handle/20.500.12840/1151/Angela_Tesis_Maestro_2018. pdf? sequence $=5$ \&isAllowed $=y$

Contreras, K. A., \& Hernández, E. (2019). Redes de apoyo familiares y feminización del cuidado de jóvenes estudiantes universitarios. Revista Hispana para el Análisis de Redes Sociales, 30(1), 54-67. http://dx.doi.org/10.5565/rev/redes.766

Diener, E. (2000). Subjective well-being: The science of happiness and a proposal for a national index. American Psychologist, 55(1), 34-43. http://dx.doi. org/10.1037/0003-066X.55.1.34

Diener, E., Emmons, R. E., Larsen, R. J., \& Griffin, S. (1985). The satisfaction with life scale. Journal of Personality Assessment, 49(1), 71-75. http://dx.doi. org/10.1207/s15327752jpa4901_13

Diener, E., Lucas, R. E., \& Oishi, S. (2002). Subjective well-being: The science of happiness and life satisfaction. In C. R. Snyder \& S. Lopez (Eds.), Handbook of positive psychology (pp. 463-473). Oxford University Press. http://dx.doi.org/10.1093/ oxfordhb/9780195187243.013.0017

Diener, E., Suh, E. M., Lucas, R. E., \& Smith, H. L. (1999). Subjective well-being: Three decades of progress. Psychological Bulletin, 125(2), 276-302. https://media. rickhanson.net/Papers/SubjectiveWell-BeingDiener.pdf

Diez, M., Morgado, B., \& González, M. M. (2016). El apoyo social y la satisfacción vital, factores clave en el caso de las madres adoptivas solas. Apuntes de Psicología, 34(2-3), 139-149. http://www.apuntesdepsicologia.es/index.php/revista/ article/view/605/451

Escobedo, M. T., Hernández, J. A., Estebané, V., \& Martínez, G. (2016). Modelos de Ecuaciones Estructurales: Características, Fases, Construcción, Aplicación y Resultados. Ciencia \& Trabajo, 18(55), 16-22. http://dx.doi.org/10.4067/S071824492016000100004

Fernández, J. M., Díez, D., Malpica, M. J., \& Hamido, A. (2010). Relación entre el apoyo social, la satisfacción vital y las expectativas de futuro de menores 
acogidos en centros de protección. Electronic Journal of Research in Educational Psychology, 8(2), 643-654. https://pdfs.semanticscholar.org/5b3d/ f4fcb681b81581b5eb922d0830613f3b9e82.pdf

García, L., Quintana-Orts, C., \& Rey, L. (2020). Cibervictimización y satisfacción vital en adolescentes: la inteligencia emocional como variable mediadora. Revista de Psicología Clínica con Niños y Adolescentes, 7(1), 38-45. http://dx.doi. org/10.21134/rpcna.2020.07.1.5

Hombrados-Mendieta, I., García, A., Gómez-Jacinto, L., \& Palma, M. O. (2016). Resiliencia, apoyo social y satisfacción vital de la población inmigrante. En D. Carbonero, E. Raya, N. Caparros, \& Ch. Gimeno (coords.), Respuestas transdisciplinares en una sociedad global: aportaciones desde el Trabajo Social (pp. 1-11). Universidad de la Rioja.

Lagos, J. L. (2019). Relaciones intrafamiliaresy satisfacción familiaren estudiantes universitarios de una institución pública [Tesis de especialidad profesional inédita, Universidad Nacional Federico Villarreal]. Repositorio Institucional UNFV. http://repositorio. unfv.edu.pe/bitstream/handle/UNFV/3748/UNFV_LAGOS_CARDENAS_\%20 JHUDYT_LILIANA_SEGUNDA_ESPECIALIDAD_2019.pdf? sequence=1\&isAllowed =y

Mallma, N. (2016). Relaciones intrafamiliares de dependencia emocional en estudiantes de psicología de un centro de formación superior. Acta Psicológica Peruana, 1(1), 107-124. http://revistas.autonoma.edu.pe/index.php/ACPP/article/view/48/36

Martos, M. J. (2016). Análisis de la satisfacción vital, salud y apoyo social en pacientes con hipertensión arterial. Revista de Psicología de la Salud (New Age), 4(1), 24-60. http://revistas.innovacionumh.es/index.php?journal=psicologiasalud\&page=arti cle\&op=view\&path\%5B\%5D=685\&path\%5B\%5D=162

Matos, G. C., Soares, M. R., Escobal, A. P. L., Quadro, P. P., \& Rodrigues, J. B. (2019). Rede de apoio familiar à gravidez e ao parto na adolescência: uma abordagem moscoviciana. Journal of Nursing and Health, 9(1), 1-9. http://dx.doi.org/10.15210/JONAH.V911.12754

Matus, R. E. (2017). Bienestar subjetivo y funcionamiento de las relaciones intrafamiliares en jóvenes universitarios [Tesis de maestría inédita, Universidad de Montemorelos]. DSpace. http://dspace.biblioteca.um.edu.mx/xmlui/bitstream/ handle/20.500.11972/359/Tesis\%20Rosa\%20Elva\%20Matus\%20S\%C3\%A1nchez. pdf? sequence $=1 \&$ is Allowed $=y$

Melguizo-Garín, A., Martos-Méndez, M. J., \& Hombrados-Mendieta, I. (2019). Influencia del apoyo social sobre el estrés y la satisfacción vital en padres de niños con cáncer desde una perspectiva multidimensional. Psicooncología, 16(1), 25-42. http://dx.doi.org/10.5209/PSIC.63646

Minuchin, S. (1986). Familias y terapia familiar. Gedisa.

Nie, Q., Tian, L., \& Huebner, E. S. (2020). Relations among family dysfunction, loneliness, and life satisfaction in Chinese children: a longitudinal mediation model. Child Indicators Research, 13(3), 839-862. http://dx.doi.org/10.1007/s12187-019-09650-6

Novoa, C., \& Barra, E. (2015). Influencia del apoyo social percibido y los factores de personalidad en la satisfacción vital de estudiantes universitarios. Terapia Psicológica, 33(3), 239-245. http://dx.doi.org/10.4067/S0718-48082015000300007 
Orozco, F. J. (2014). Hacia una mejor comprensión de la satisfacción vital percibida: una revisión de la literatura sobre los factores asociados a la satisfacción vital de los inmigrantes [Tesis de maestría inédita, Universitat Jaume 1]. Repositori Universitat Jaume I. http://repositori.uji.es/xmlui/bitstream/handle/10234/113605/TFM_ Orozco_Dopico_Francisco.pdf?sequence=1\&isAllowed =y

Pinazo-Hernandis, S., Torregrosa-Ruiz, M., Jiménez-Martí, M., \& Blanco-Molina, M., (2019). Participación social y satisfacción vital: diferencias entre mujeres y hombres mayores. Revista de Psicología de la Salud, 7(1), 202-234. http://revistas. innovacionumh.es/index. .php?journal=psicologiasalud \&page=article\&op=view \&path\%5B\%5D =1501\&path\%5B\%5D =616

Reina, M. C., \& Oliva, A. (2015). De la competencia emocional a la autoestima y satisfacción vital en adolescentes. Behavioral Psychology/Psicología Conductual, 23(2), 345359. https://www.researchgate.net/publication/285199595_De_la_competencia_ emocional_a_la_autoestima_y_satisfaccion_vital_en_adolescentes

Rivera, M. E., \& Andrade, P. (2010). Escala de evaluación de las Relaciones Intrafamiliares (E.R.I.). Uaricha Revista de Psicología, 14, 12-29. https://www.academia. edu/6596473/Escala_de_evaluaci\%C3\%B3n_de_las_Relaciones_Intrafamiliares

Ruiz, E. M. (2019). Relaciones intrafamiliares y estrés cotidiano en estudiantes de primaria de una institución educativa de Virú [Tesis de maestría inédita, Universidad César Vallejo]. DSpace. http://repositorio.ucv.edu.pe/bitstream/handle/UCV/37874/ ruiz_ce.pdf? sequence $=1 \&$ isAllowed $=y$

Ruiz, M. A., Pardo, A., \& San Martín, R. (2010). Modelos de ecuaciones estructurales. Papeles del Psicólogo, 37(1), 34-45. https://www.redalyc.org/pdf/778/77812441004.pdf

Schoeps, K., Ordóñez, A., Montoya, I., \& Barrón, R. (2014). Funcionamiento familiar, personalidad y satisfacción vital en las parejas casadas. International Journal of Developmental and Educational Psychology, 1(6), 381-392. http://dx.doi. org/10.17060/ijodaep.2014.n1.v6.758

Secretaría de Salud (1987). Reglamento de la Ley General de Salud en Materia de Investigación para la Salud. Porrúa.

Seligman, M. (2003). La Auténtica Felicidad. (M. D. Esteva \& D. Cabeza, Trad.). Ediciones B.

Torre, M. J., Casanova, P. F., Villa, M., \& Cerezo, M. T. (2013). Consistencia e inconsistencia parental: relaciones con la conducta agresiva y satisfacción vital de los adolescentes. European Journal of Education and Psychology, 6(2), 135-149. http:// dx.doi.org/10.1989/ejep.v6i2.112

Urbano, A., Álvarez, L., \& Iglesias, M. T. (2018). Adaptabilidad y cohesión familiar del alumnado de Educación Secundaria Obligatoria. Aula Abierta, 47(2), 237-244. http://dx.doi.org/10.17811/rifie.47.2.2018.237-244

Vallejo-Martín, M., \& Moreno, M.-P. (2016). Satisfacción vital y su relación con otras variables psicosociales en población española residente en Alemania. Escritos de Psicología, 9(2), 12-21. http://dx.doi.org/10.5231/psy.writ.2016.2803

Veenhoven, R. (1994). El estudio de la satisfacción con la vida. Intervención Psicosocial, 3(9), 87-116. http://www.copmadrid.org/webcopm/publicaciones/ social/1994/vol3/arti8.htm 
Veloso, C., Muñoz, N., Palza, A., Roa, C., Tapia, C., \& Lee, S. (2015). Asociación de inteligencia emocional, satisfacción vital con conducta prosocial en jóvenes escolarizados de la XV región. Límite. Revista Interdisciplinaria de Filosofía y Psicología, 10(34), 50 58. https://dialnet.unirioja.es/servlet/articulo?codigo $=6612108$

Villafrade, L. A., \& Franco, C. M. (2016). La familia como red de apoyo social en estudiantes universitarios que ingresan a primer semestre. Espiral, revista de Docencia e Investigación, 6(2), 79-90. http://dx.doi.org/10.15332/erdi.v6i2.1651

Zaquinaula, A., \& Murillo, L. G. (2015). Relaciones intrafamiliares y bienestar psicológico en adolescentes estudiantes del nivel secundario de la ciudad de Ayabaca, 2015 [Tesis de maestría inédita, Universidad Privada Antonio Guillermo Urrelo]. Repositorio UPAGU. http://repositorio.upagu.edu.pe/bitstream/handle/UPAGU/181/MURILLO\%20Y\%20 ZAQUINAULA.pdf?sequence=1\&isAllowed $=y$ 